\title{
Inflammasomes contributing to inflammation in arthritis
}

\author{
Authors: \\ Lotte Spel ${ }^{1}$ and Fabio Martinon ${ }^{1 *}$ \\ Affiliations: \\ ${ }^{1}$ Departement of Biochemistry, University of Lausanne, Epalinges 1066, Switzerland. \\ *Correspondence to: \\ Fabio Martinon, \\ Departement of Biochemistry \\ University of Lausanne, \\ Ch. Des Boveresses 155 \\ Epalinges 1066, Switzerland \\ Phone: +41-21-692.5695 \\ Fax: +41-21-692.5705 \\ Email: Fabio.Martinon@unil.ch
}

Conflicts of interest statement: The author discloses no conflicts of interest.

Running title: inflammasomes in arthritis

Keywords : inflammasome, gout, arthritis, NLRP3, innate immunity, CARD8

Words count: 11300

Number of Figures: 3

Number of Tables 1 


\section{Summary}

Inflammasomes are intracellular multiprotein signaling platforms that initiate inflammatory responses in response to pathogens and cellular damage. Active inflammasomes induce the enzymatic activity of caspase-1 resulting in the induction of inflammatory cell death, pyroptosis, and the maturation and secretion of inflammatory cytokines IL-1 $\beta$ and IL-18. Inflammasomes are activated in many inflammatory diseases, including autoinflammatory disorders and arthritis, and inflammasome-specific therapies are under development for the treatment of inflammatory conditions. In this review, we outline the different inflammasome platforms and recent findings contributing to our knowledge about inflammasome biology in health and disease. In particular we discuss the role of the inflammasome in the pathogenesis of arthritic diseases including rheumatoid arthritis, gout, ankylosing spondylitis and juvenile idiopathic arthritis, and the potential of newly developed therapies that specifically target the inflammasome or its products for the treatment of inflammatory diseases. 


\section{INTRODUCTION}

Inflammasomes are molecular complexes formed within the cytosol of innate immune cells

1. Upon activation, these signaling platforms acquire a proteolytic activity that initiates inflammatory events such as the maturation of the pro-inflammatory cytokine interleukin $1 \beta(\mathrm{IL}-1 \beta)^{2}$. Inflammasomes can detect various insults including the presence of pathogens and tissue damage ${ }^{3}$. While the main physiological function of inflammasomes is to elicit an immune response and to contribute to the maintenance of tissue homeostasis and repair, deregulated inflammasome activation can be harmful. Several inflammasomes are directly linked to hereditary and acquired autoinflammatory diseases ${ }^{4}$. The clinical importance of inflammasomes has also been demonstrated in diseases with a more complex etiology characterized by inflammation and tissue damage. Excess inflammasome activation has been shown to contribute to cancer, metabolic disorders including type 1 and type 2 diabetes, neurodegenerative diseases and autoimmunity such as in systemic lupus erythematosus (SLE). Increased inflammasome response has also been suggested to be a hallmark of the aging process ${ }^{5}$.

Several pathologies of the joint are linked to aberrant inflammasome engagement. This was investigated in patients with gout, rheumatoid arthritis (RA), and juvenile idiopathic arthritis (JIA) ${ }^{6}$.

Understanding the mechanisms leading to the activation of inflammasomes in physiological and pathological conditions, will ameliorate the diagnosis and treatment of aberrant inflammation including in diseases with arthritis. Extensive efforts have been deployed in the last two decades to characterize how the different inflammasomes are activated or regulated. Several key discoveries have dramatically improved our 
understanding of these pathways and led to the development of new therapeutic strategies aimed at targeting downstream mediators. Drugs that could directly target inflammasome assembly are being developed and could provide valuable tools to dampen inflammation in diseases with aberrant inflammasome activation. In this review we discuss major advances that improved our understanding of inflammasomes biology. We will also highlight several key questions that are still open. In particular, we will focus on how the different inflammasomes contribute to different arthritic disease.

\section{NLRs function as sensors of pathogen and cellular perturbations}

Classical inflammasome pathways are engaged by the oligomerization of patternrecognition receptors (PRR) following direct or indirect recognition of pathogen effectors or perturbations in cellular homeostasis (Figure 1). Among these receptors, the nucleotidebinding leucine-rich repeat receptors (NLRs) were among the first characterized. These proteins are typical PRR that can assemble signaling platforms with specific enzymatic activities, often referred under the generic name of signalosomes ${ }^{7}$. Several NLRs such as NLRP3 can form molecular structures harboring the enzymatic properties of typical inflammasomes. The generic modular organization of NLR proteins is formed by a conserved tripartite domain structure consisting of a N-terminal recruiting domain, a central nucleotide-binding domain that is capable of oligomerization, and a C-terminal leucine rich repeat (LRR) domain that regulates the assembly of the complex. This modular organization is found in several innate immune pathways in mammals as well as in plants where they form the largest family of defense molecules ${ }^{8,9}$. While plant and mammalian NLRs are structurally and functionally very similar, they are believed to be the product of 
convergent evolutions ${ }^{10}$, indicating that this successful modular architecture may underline a possible conserved strategy of cellular host defenses.

The central conserved region of plant and mammalian NLRs consists of a NTPase domain. This domain is found in a widespread family of signal transduction ATPases with numerous functions that includes the apoptosome protein Apaf-1 ${ }^{11}$. The NTPase domain is believed to function as a very rapid molecular switch', that cycles between an adenosine 5'-diphosphate (ADP)-bound inactive state and an adenosine 5'-triphosphate (ATP)-bound active state ${ }^{12}$. Upon engagement, Apaf-1 oligomerizes to assemble an apoptosome complex. Similarly, NLRs assemble specific signalosomes such as inflammasomes. Both active inflammasomes and the apoptosome recruit caspases that will provide proteolytic effector functions. The apoptosome recruits directly the proapoptotic caspase-9. Similarly, the NLRC4 protein recruits directly the inflammatory caspase, caspase-1, while other NLRs such as NLRP3 recruit caspase-1 indirectly via the adaptor protein ASC ${ }^{13}$ (Figure 1A).

The NLRP3 inflammasome can be activated by a wide variety of stimuli, apparently unrelated. It can detect pathogens, including bacteria and viruses, as well signals released by exposure to damaging particles or drugs thereby eliciting a sterile inflammation. Most NLRP3 activators trigger a form of cellular stress that correlates with changes in ion content of the cell. A typical hallmark of NLRP3 activation is the efflux of potassium ions $\left(\mathrm{K}^{+}\right)^{14}$, a phenomenon that is also required for the assembly of the apoptosome ${ }^{15}$. Another feature of NLRP3 activation is the disruption of the trans-Golgi network (TGN) ${ }^{16}$. NLRP3 activation was also observed upon disruption of other organelles such as lysosomal rupture, and mitochondrial malfunction (reviewed in ${ }^{17}$ ). In response to various stimuli, NLRP3 
translocates to disrupted TGN leading to inflammasome formation ${ }^{16}$. This, suggests that TGN disruption could be the central sensing mechanism of NLRP3 activation possibly downstream of signals emerging from damaged mitochondria or lysosomes.

The pro-inflammatory role of NLRP3 was initially demonstrated by the discovery of its role in promoting cryopyrin-associated periodic syndrome (CAPS) ${ }^{18}$. CAPS is an inherited autoinflammatory disorder characterized by systemic inflammation with fever often presenting with skin rashes and central nervous system inflammation. While arthralgia is common in patients with CAPS, arthritis is less frequent ${ }^{19}$. Inflammation in CAPS is caused by gain-of-function mutations in NLRP3 leading to aberrant inflammasome activation and direct initiation of an inflammatory cascade ${ }^{20}$. These findings demonstrated that inflammasome activation per se was sufficient to initiate a full-blown systemic inflammation in humans.

The NLRP1 inflammasome differs from other NLRs by the addition of a C-terminal extension made of a FIIND domain and a CARD. Caspase-1 is mainly recruited to NLRP1 through interactions with the C-terminal CARD (Figure 1A). In the process of activation, the FIIND undergoes self-cleavage, an event that is required for activation. NLRP1 responds to the enzymatic activities of pathogen effectors such as the Lethal Toxin (leTox) from Bacillus anthracis ${ }^{21}$ as well as by non-pathogen-associated triggers such as the drug Val-boro Pro, an anti-cancer molecule that inhibits the cytosolic serine proteases Dpp8 and Dpp9 ${ }^{22,23}$.

Gain of function mutations within NLRP1 have bene described in humans to cause uncontrolled inflammasome activation promoting a familial autoinflammatory skin disease associated with cancer ${ }^{24}$. Other mutations within NLRP1 have been linked to 
autoinflammation with arthritis and dyskeratosis (AIADK) also known as NAIAD (NLRP1-associated autoinflammation with arthritis and dyskeratosis) ${ }^{25}$. Moreover, polymorphisms in NLRP1 were found to be associated with immune disorders such as vitiligo and rheumatoid arthritis ${ }^{26}$, further highlighting the importance of this pathway in humans as a host defense pathway whose alteration can predispose to autoimmune or autoinflammatory diseases ${ }^{27}$.

CARD8 is a caspase recruitment domain-containing protein, also known as TUCAN or Cardinal, which is structurally very similar to NLRP1 Although its N-terminus is much shorter than that of NLRP1, CARD8 also contains a FIIND domain and a CARD at its Cterminus. CARD8 was initially discovered as a $48 \mathrm{kDa}$ protein expressed in various tissues and cell types ${ }^{28}$. To date, up to five mRNA isoforms have been described that encode for CARD8 proteins with divergent $\mathrm{N}$-terminal but equal C-terminal regions ${ }^{29}$. CARD8 does not respond to pathogen effectors including Lethal Toxin therefore CARD8 was considered to be a decoy NLR which may inhibit inflammasome activation. Interestingly, CARD8 inhibits NLRP3 inflammasome activity but not NLRP1. In fact, similar to NLRP1, CARD8 activates caspase- 1 and induces pyroptosis when cells were treated with Val-boro Pro ${ }^{30}$. CARD8 knock-down resulted in enhanced IL-1 $\beta$ secretion when using NLRP3 activating stimuli. Furthermore, CARD8 was unable to bind to CAPS-associated mutant NLRP3 possibly contributing to the overactive NLRP3 inflammasome in these patients ${ }^{31}$. CARD8 has evolved to play a role in the delicate balance of activating and inhibiting signals that determine NLRP3 inflammasome activity. Disturbance of such balance may contribute to the development of inflammatory diseases which is probably why CARD8 polymorphisms are often associated with risk for arthritic diseases ${ }^{32-36}$. 
Other NLR inflammasomes including the NLRC4 and NLRP12 inflammasome have been linked to autoinflammatory diseases (review in ${ }^{13}$ ). Inflammasomopathies are defined as inflammasome-related diseases. These rare conditions appear to have a wide spectrum of phenotypes that make often the diagnosis challenging. Increased awareness of these rare diseases has facilitated the identification of several inflammasomes, including some that could contribute to inflammation in complex diseases such as arthritis.

\section{Non-NLR inflammasomes}

In addition to NLRs other innate immune sensors have evolved to form inflammasomes (Figure 1B). Among these, the Pyrin inflammasome is the best understood. Pyrin was initially identified as the product of the $M E F V$ gene involved in Familial Mediterranean fever (FMF) ${ }^{37,38}$.

FMF is the most frequent monogenic autoinflammatory condition described so far. It differs from other inflammasomopathies in that it is inherited mostly in an autosomal recessive fashion, despite the fact that in FMF patients, systemic inflammation is caused by a gain of function in the pyrin protein causing aberrant activation of the pyrin inflammasome ${ }^{39}$. It is not known why patients with only one allele mutated only rarely show signs of FMF. It could indicate a dose effect of MEFV mutations in FMF or that the presence of the natural allele dampens constitutive pyrin inflammasome formation in heterozygous carriers.

Several musculoskeletal symptoms are present in FMF, including arthralgia and arthritis. Moreover, in individuals without apparent signs of FMF but harboring a heterozygous mutation in the $M E F V$ gene, increased prevalence of seronegative rheumatoid arthritis was

observed ${ }^{40}$. Evidences also indicate that the $M E F V$ gene might participate in the 
pathogenesis of systemic onset juvenile idiopathic arthritis ${ }^{41}$. These findings also revealed that gain of function in one of the $M E F V$ alleles is sufficient to increase the risk of autoinflammatory arthritis, further indicating that the pyrin inflammasome may contributes to joint inflammation.

The AIM2 inflammasome may also contribute to arthritis. The protein AIM2 detects free DNA. It assembles an inflammasome in the context of released viral and bacterial DNA as well as self-DNA exposed by damaged cells (review $\mathrm{in}^{42}$ ). In mice, using a model of arthritis caused by deficiency in DNAse II, AIM2 inflammasome activation was found to contribute to joint inflammation ${ }^{43,44}$. Additional studies are required to investigate whether tissue damage or infections in the joint may trigger AIM2, thereby contributing to inflammation in human arthropathies.

\section{Inflammatory signals triggered by inflammasomes activation}

Inflammasome assembly originates from the activation of different platforms that respond to specific stimuli. Despite this complexity that probably reflect an evolutionary race aimed at the sensing of harmful conditions, all inflammasomes converge to the activation of one enzymatic function; the activation of inflammatory caspases, mainly caspase-1. Two main consequences result from caspase-1 activation: cell death and cytokine release.

IL-1 $\beta$ and IL-18 are the two cytokines that rely the most on caspase-1 activation. Both these cytokines are synthetized as precursors that require proteolytical cleavage to be fully active. Caspase-1 is the main protease for IL-1 $\beta$ and IL-18 maturation, therefore these two inflammatory mediators are typical products of inflammasome activation capable of communicating the inflammatory signal onto cells harboring IL-1 $\beta$ and/or IL-18 receptors 
(Figure 2). Several inflammasomopathies can be efficiently treated with drugs that block these cytokines, particularly IL-1 $\beta$. This is particularly evident in CAPS patients, where treatment with biologics that block signaling by the IL-1 receptors dampens most signs of systemic inflammation ${ }^{45}$.

Another consequence of inflammasome assembly and caspase- 1 activation is the induction of pyroptosis. Pyroptosis is a pro-inflammatory form of cell death that is associated with the disruption of cellular membranes leading to the extracellular release of cytosolic content. IL-1 $\beta$ and IL-18 are synthetized in the cytosol and do not carry leader sequences to target them to the secretory pathway. These cytokines cannot be released by the "classical" pathway of secretion. In contrast the disruption of plasma membrane integrity observed upon inflammatory caspase activation may present the main mechanism by which these cytokines are released following proteolytic maturation ${ }^{46}$. While cell death could be a prerequisite for the release of IL-1 $\beta$ and IL-18, not all cells commit to pyroptosis upon inflammasome assembly ${ }^{47,48}$. Therefore, alternative pathways of IL-1 $\beta$ and IL-18 secretion may exist. The identification of GSDMD as a cytosolic caspase-1 substrate, has shed some light on the process of pyroptosis and cytokine release ${ }^{49,50}$. Upon cleavage GSDMD releases an N-terminal fragment that oligomerizes and forms pore-like structures within the membrane lipid bilayer. These pores have been proposed to function as conduits for the release of small proteins including IL-1 $\beta$ and IL-18 ${ }^{51,52}$.

The formation of GSDMD pores has also been reported to disrupt membrane integrity ${ }^{49,50}$, a process that can trigger an adaption program aimed at repairing the damage ${ }^{53}$. If the repair is unsuccessful or the damage too sizeable the GSDMD pores allow for complete lysis of the cell and death by pyroptosis ${ }^{46}$. 
While pyroptosis has been studied extensively in mice and in the context of infection with intracellular bacteria, its role in human inflammatory and autoimmune diseases is still unclear. The identification of markers and assays to monitor key features of pyroptosis in human tissues will help address the role of this process in humans. In particular it would be of interest to identify proinflammatory mediators released by damaged cells beyond IL$1 \beta$ and IL-18. It is likely that additional cytosolic components release during pyroptosis may contribute to aspect of inflammation and autoimmunity in damaged tissues.

\section{Cell types competent for inflammasome activation}

Inflammasome activation is a multi-step process that is tightly controlled. Most cell types need to be primed to become competent for inflammasome activation in vitro. This priming, also known as signal 1 , is provided by treatment with strong proinflammatory stimui such as lipopolysaccharides (LPS) or phorbol myristate acetate (PMA), two molecules that trigger NFKB activation (Figure 2). It was postulated that these signals may function by promoting the induction of inflammasome components. Signal 1 may also promote postranslational modifications of inflammasome components required for activation ${ }^{17}$. However, the exact nature of signal 1 and its function is still debated. Importantly, little is known about the signals that make cells competent for inflammasome activation in vivo in humans. The importance of signal 1 is illustrated in patients with gain of function mutations within inflammasome platforms such as in CAPS. While myeloid cells from these patients have constitutive activation of the NLRP3 protein, actual inflammasome assembly is only observed upon treatment of these cells with LPS ${ }^{20}$. In these patients, yet unidentified signals may provide the signal 1 that contribute to IL-1 $\beta$ mediated systemic inflammation. Because there is no clear evidence that infectious events 
may initiate inflammatory flares in CAPS, signal 1 may originate from endogenous processes such as low-grade inflammation involved tissue repair and homeostasis ${ }^{54}$. Understanding the exact nature of signal 1 in patients with inflammasomopathies may shed some light on the physiological setting that contributes to inflammasome activation upstream of its assembly.

It is likely that the nature of signal 1 will differ between cell types and the different inflammasome platforms. The context of inflammasome activation is therefore key and may contribute to the development of inflammasome related diseases. Inflammasomes are mostly studied in cells of myeloid origin such as monocytes, macrophages, neutrophils, and dendritic cells. These cell types show different responses to inflammasome stimuli, for example, neutrophils are less susceptible to pyroptosis than macrophages ${ }^{47}$. In mice it was shown that differentiation protocols used to produce dendritic cells and macrophages determine the response to inflammasome inducers, further demonstrating how context affects inflammasome activation and the difficulty to extrapolate the nature and contribution of signal 1 from in vitro studies to physiological or pathological conditions ${ }^{55}$. While poorly understood, inflammasome activation can also occur in non-phagocytic cells, such as $\mathrm{T}$ cells, endothelial cells, and epithelial cells. For example, in immune and nonimmune cells of the gut, inflammasome activation such as the NLRP6 and NLRC4 inflammasomes contribute to the maintenance of gut microbiota and gut-brain homeostasis 56. In T cells, the AIM2-like protein IFI16 has been shown to detect products from HIV infection to promote $\mathrm{T}$ cell pyroptosis ${ }^{57}$. In another study it was proposed that the complement cascade can engage the NLRP3 inflammasome in human T cells to direct $\mathrm{T}$ cells differentiation towards Th1 lineage ${ }^{58}$. The $\mathrm{T}$ cell inflammasome has also been 
suggested to contribute to autoimmunity. Studies in mice have shown a role for the inflammasome in promoting Th17 differentiation in a mouse model of autoimmune encephalomyelitis ${ }^{59}$.

Different inflammasome platforms have evolved to protect against pathogens and perturbations of cellular homeostasis. Inflammasomes aim for restoration of damage by initiating immune responses and repair mechanisms. When activation of inflammasome platforms is not in balance, its inflammatory effects contribute to unwarranted and damaging reactions such as the inflammation observed in arthritic diseases including RA and gout.

\section{Inflammasome in rheumatoid arthritis}

The systemic autoinflammatory disease characteristic for rheumatoid arthritis (RA) primarily affects joints and is driven by chronic synovial inflammation leading to irreversible cartilage degradation and joint destruction ${ }^{60,61}$. With approximately $1 \%$ of the population affected worldwide ${ }^{62}$, RA is among the most common inflammatory diseases and therefore an active topic of investigation. The involvement of inflammasomes in the pathogenesis of RA has been demonstrated in various studies in animal models, in genetic association studies as well as in samples from patients.

In search for specific biomarkers for RA, the cytokine levels in sera and synovial fluid of RA patients have been investigated. Increased concentrations of many inflammatory cytokines were found in RA sera when compared to healthy controls, most prominently TNF, IL- 6 and IL- $1 \beta^{63}$. In particular the IL-1 $\beta$ of both serum and synovial fluid increased 
in active disease and was associated with higher IL-18 levels in the synovial fluid ${ }^{63}$. This argues for a prominent role of the inflammasome in the pathogenesis of RA (Table 1).

Dampening of essentially all canonical inflammasomes, by gene knock-out of ASC, protected mice from developing arthritis in a collagen-induced arthritis model ${ }^{64}$, and attenuated arthritic symptoms in an antigen-induced arthritis model ${ }^{65}$. In contrast, gene knock-out of either NLRP3 or caspase-1 rendered the mice susceptible to arthritis induction in both models. These results are surprising given that in following years multiple studies using animal models of arthritis showed a significant role for NLRP3 inflammasome ${ }^{6}$. These differences may reveal the involvement of multiple inflammasomes in RA whose function depends on the context and the presence of specific signal 1 promoting conditions. NLRP3 role in RA is supported by several observations. The severity of arthritis clinical score showed a positive correlation with NLRP3 levels in synovial tissue ${ }^{66}$ and clinical features of arthritic disease could be inhibited by suppression of the NLRP3 inflammasome in macrophages ${ }^{67}$. Furthermore, in an adjuvant-induced arthritis model in rats, upregulation of NLRP3 inflammasome components was observed in fibroblast-like cells isolated from the synovium ${ }^{68,69}$. These results are in line with results obtained in mice lacking the A20/Tnfaip3 gene which predispose them to a RA-like disease. In this animal model of RA, the expression of NLRP3 inflammasome components are enhanced in macrophages and NLRP3 deficiency suppressed arthritis development and cartilage destruction ${ }^{70}$. In addition, deficiency of caspase-1 or IL-1 receptor diminished RAassociated inflammation and joint destruction in this study. The importance of IL-1 was also demonstrated in a mouse model of chronic arthritis, in which cell wall fragments of Streptococcus pyogenes are injected into the joint to induce inflammation ${ }^{71}$. Mice deficient 
for IL-1 $\beta$ were protected from progressing to chronic arthritis ${ }^{71,72}$. Moreover, IL-1 deficiency was shown to protect against antibody-induced arthritis as well ${ }^{73}$.

In human studies, evidence also exists that supports a role for NLRP3 inflammasome pathway in RA. Gene expression of NLRP3, ASC, caspase-1, IL-1 $\beta$ and IL-1R were significantly higher in PBMCs of RA patients compared to healthy controls ${ }^{34,74-76}$ as well as the secretion of IL-1 $\beta^{74,77}$. Single nucleotide polymorphisms at the NLRP3 locus are associated with RA susceptibility ${ }^{34,75}$ and correlate with higher disease activity ${ }^{78}$. A previously found $N L R P 3$ polymorphism was shown not to associate with RA susceptibility by itself, however combined with a polymorphism within $C A R D 8$, a possible regulator of the inflammasome, the NLRP3 polymorphism associated with RA susceptibility and severity ${ }^{33}$. In addition, CARD8 expression was lower in PBMCs of RA patients compared to healthy controls ${ }^{34}$. Similar to PBMCs, cells directly isolated from the synovial fluid showed increased expression of inflammasome genes ${ }^{79}$. In particular myeloid and endothelial cells showed enhanced expression of NLRP3, ASC and caspase-1 and were proposed to be the primary source of IL- $1 \beta$ secretion in the synovium ${ }^{80}$.

Inflammasome activity results in maturation of IL-1 $\beta$, a cytokine heavily implicated in RA pathogenesis, but also IL-18. High levels of IL-18 in patients sera and synovial fluid have been described ${ }^{63,81,82}$ and polymorphisms in the IL-18 gene locus were associated with increased risk for RA ${ }^{83}$. Animal studies suggest that IL-18 contributes to collagen-induced arthritis in rats ${ }^{84}$ as well as in a mouse models of RA elicited by treatment with the TLR2 agonist zymosan ${ }^{85}$. On a functional level, IL-18 was shown to promote chemotaxis of monocytes to the synovium ${ }^{85}$ and angiogenesis within the arthritic joint ${ }^{86}$. Moreover, IL- 
18 was also able to induce fibroblast-like synoviocytes to secrete pro-osteoclastic cytokines suggesting a role in bone resorption ${ }^{87}$.

Finally, a single NLRP1 gene polymorphisms was identified as a risk factor for RA ${ }^{88}$. This particular polymorphism resulted in increased expression of NLRP1. In an adjuvantinduced arthritis model in rats increased NLRP1 expression was also observed ${ }^{89}$, and inhibition of NLRP1 inflammasome ameliorated the symptoms of arthritis in AIA rats as

well as CIA mice ${ }^{89-91}$. The prominent role of NLRP1 inflammasome in CIA mice may explain why NLRP3 deficiency had limited effect on arthritis induction in this animal mode. While the pathways and mechanisms leading to inflammasome activation is RA are still poorly understood, the above studies clearly show the involvement of the inflammasome platform and its downstream cytokines IL-1 $\beta$ and IL-18 in RA disease. Inflammasome-targeted therapies may therefore benefit RA patients and will be discussed below.

\section{Inflammasome in gouty arthritis}

Deposition of monosodium urate (MSU) crystals or calcium pyrophosphate dehydrate (CPPD) crystals in the joint underlie the development of gout or pseudogout, respectively. Characteristic for these autoinflammatory diseases are the flares of inflammation, causing fever as well as severe pain and swelling of the joint(s). Since gout has a clear cause, detection of crystals in the synovial fluid is adequate for diagnosis and the demand for large studies to identify biomarkers remained inexistent. Rather, studies focused on identifying risk factors for the disease and cellular mechanisms by which crystals evoke inflammatory reactions. 
Several SNPs in inflammasome-relates gene loci were identified as risk factor for gout, including NLRP3 ${ }^{92}$, IL-1 $\beta^{93}$ and CARD8 ${ }^{36}$ (Table 1). Moreover, genetic variants of PPARGC1B (peroxisome proliferator-activated receptor-y coactivator 1a) that resulted in increased expression of NLRP3 and IL-1 $\beta$ in PBMCs were significantly associated with gout ${ }^{94}$. High levels of NLRP3, caspase-1, IL-1 $\beta$, and IL-18 were detected in patient sera and/or synovial fluid ${ }^{92,95-97}$. IL-1 signalling, as a result of inflammasome activation, is a very efficient signal 1 that can increase the expression of inflammasome components, therefore increased expression of inflammasome components may indicate the presence of an active inflammasome in these patients.

Evidence from inflammasome-deficient mice supports a role for the inflammasome complex in gout pathology. Murine models of crystal-induced inflammation mimic the complex interplay that occurs between resident and infiltrating immune cells in an inflamed joint by injection of MSU crystals into the joint or within the peritoneal cavity ${ }^{98}$. Accordingly, neutrophil infiltration towards the MSU injection site was reduced in the absence of functional NLRP3, ASC, or caspase-199,100. Furthermore, MSU-induced secretion of IL-1 $\beta$ was prevented in macrophages lacking NLRP3, ASC or caspase-1 ${ }^{100,101}$. Similar results were obtained using CPPD crystals ${ }^{100}$. Interestingly, inhibition of inflammation was observed to be more robust and sustained in IL-1R deficient animals compared to ASC or caspase-1 deficient animals ${ }^{100}$, indicating that in absence of inflammasomes pathogenic microcrystals can activate an alternative pathway of IL-1 activation. This could be mediated by IL-1 $\alpha$ or may involve other proteolytic event that could promote IL-1 $\beta$ maturation such as the release of neutrophil serine proteases ${ }^{72}$. 
MSU crystals were found to activate the complement cascade leading to active complement subunits $\mathrm{C} 3 \mathrm{a}$ and $\mathrm{C} 5 \mathrm{a}{ }^{102,103}$. Specifically, C5a induced neutrophil infiltration towards the site of injection and activated NLRP3 inflammasome in peritoneal macrophages through ROS production ${ }^{103}$. Inhibition of C5a resulted in diminished IL-1 $\beta$ release ${ }^{102}$. Interestingly, C5a was also found to promote NLRP3 activation in $\mathrm{T}$ cells ${ }^{58}$, indicating that this mechanism of NLRP3 activation is relevant beyond myeloid cells and microcrystal-induced inflammation.

In vitro, the addition of MSU per se did not induce inflammasome activation in murine BMDM or human PBMC ${ }^{104,105}$ and was shown to require a prior priming (signal 1) of the cells. Various stimuli are able to prime monocytes/macrophages for inflammasome activation by pathogenic crystals. Indeed, priming with serum amyloid A (SAA), TLR ligands, or TNF warranted the capacity of MSU to activate the inflammasome and induce release of IL-1 $\beta$ in human cells ${ }^{104-108}$. Of interest, also soluble uric acids were able to deliver the priming signal ${ }^{109,110}$ however the precipitation of uric acid into MSU crystals was necessary for NLRP3 inflammasome activity ${ }^{110}$. These results suggest that hyperuricemia, the condition of elevated systemic uric acid levels which precedes gout pathology, may provide the priming signal that sets of inflammasome activation once MSU crystals are formed and deposited.

\section{Inflammasome in spondyloarthritis}

This type of arthritis, often called ankylosing spondylitis (AS), primarily affects the spinal and sacroiliac joints and is characterized by vertebral fusions and bone erosions that cause severe and chronic pain ${ }^{111,112}$. Of the inflammasome-related genes, those belonging to the 
IL-1 family have been implicated in the pathogenesis of AS. Polymorphisms in the $I L-1 \alpha$ or $I L-1 \beta$ gene locus associated with susceptibility for AS ${ }^{113-117}$. Furthermore, a genetic variant of the IL-1RN allele was significantly increased in AS patients compared to healthy controls ${ }^{115}$. Increased levels of IL-1 $\beta$ were measured in PBMCs of AS patients and active caspase-1 could be detected in AS synovial fluid ${ }^{97,118}$. Although caspase-1 activity and IL1 release suggest a role for the inflammasome in AS, functional data is lacking to determine a biological relevant role for the inflammasome in this disease. A recent study comparing gene expressions between PBMCs of AS patients and healthy controls showed increased expression of NLRP3, ASC, caspase-1 and IL-1 $\beta$ in AS ${ }^{119}$, yet polymorphisms in the NLRP3 region were not associated with the risk of AS ${ }^{120}$. In contrast, the C10X polymorphisms of CARD8 was found to be associated with a decreased risk of AS ${ }^{120}$.

\section{Inflammasome in pediatric arthritis}

The clinical spectrum of juvenile idiopathic arthritis (JIA) ranges from oligoarthritis with four or less joints affected, to polyarticular arthritis having more joints affected and higher damage, to systemic arthritis that affects many joints and also includes skin rashes and fever attacks (systemic-onset JIA; soJIA). Many genetic studies in JIA have been performed, but only a few studies link inflammasome to JIA pathogenesis. In a Taiwanese population a polymorphism in NLRP3 was associated with increased risk for oligo/polyarticular arthritis ${ }^{35}$. A polymorphism in IL-1Ra, a negative regulator if IL-1 signaling, was found associated with JIA patients in an Iranian population, however it was not specified which type of JIA was involved ${ }^{121}$. Cytokine profile analysis of blood plasma revealed no differences in any of the inflammasome-activated cytokines IL-1 $\alpha$, IL-1 $\beta$ or 
IL-18 between patients and healthy controls ${ }^{122}$ suggesting a modest role for the inflammasome in oligo/articular JIA pathology.

Systemic-onset JIA (soJIA) differs from other JIA types in pathogenesis. Its clinical outcome is more severe than other juvenile arthritis types and patients are at additional risk for developing macrophage activation syndrome (MAS) ${ }^{123}$. Inflammasome-activated cytokines IL-1 $\beta$ and IL-18 play a significant role in soJIA. When serum of soJIA patients was added to healthy donor PBMCs, upregulated gene expression of IL-1 $\beta$ and IL1R was observed and secretion of IL-1 $\beta$ was detected ${ }^{124}$. Direct stimulation of patient PBMCs also resulted in high levels of IL-1 $\beta$ release which was not observed in healthy donor PBMCs ${ }^{124}$. IL-18 serum as well as synovial fluid levels were markedly increased in soJIA and were shown to have a high predictive value for disease activity ${ }^{122,125}$. Moreover, IL-18 may be indicative of risk to develop MAS ${ }^{126}$.

Because of its atypical clinical presentation, soJA is classified as an autoinflammatory rather than autoimmune disorder. Symptoms of classical autoinflammatory diseases including CAPS and FMF overlap with soJIA including recurrent fever episodes, skin rashes, joint swelling and systemic inflammation ${ }^{127}$. This suggest a possible role for inflammasome dysfunction in soJIA.

Further evidence for the contribution of inflammasome biology in rheumatic diseases comes from the efficacy of inflammasome-targeted therapies used in rheumatic patients. Over the years, treatment of rheumatic diseases has evolved from complete suppression of inflammation with medications like glucosteroids to targeting of specific inflammatory pathways using specific pathway inhibitors and refined biological therapies. 


\section{Inflammasome-targeted therapies in arthritic diseases}

The prominent role for inflammasomes in different diseases including monogenic autoinflammatory diseases and more complex inflammatory disorders have provided strong interest in the identification and development of strategies to decrease inflammasome responses. Two strategies have been considered (Figure 3). The first approach aims at targeting the inflammasome specific cytokines IL-1 $\beta$ and IL-18. The second strategy aims at directly inhibiting specific inflammasome components.

\section{IL-1/IL-18 inhibitors}

Anakinra is a recombinant version of the naturally occurring endogenous IL-1ra, which is regularly used in the treatment of RA and autoinflammatory diseases. IL-1ra binds to the IL-1 receptor complex (IL-1R) without triggering a cellular signaling cascade. This binding results in competitive inhibition of the proinflammatory effects of IL-1. Anakinra has been used in RA for almost two decades (Table 1). Alternatives to Anakinra include Rilonacept

a recombinant protein which function as a soluble decoy to prevent activation of IL-1RI ${ }^{128}$ and Canakinumab, a human monoclonal antibody targeting IL-1 $\beta$. This antibody was approved for CAPS ten years ago and turned out to be very effective at inhibiting its target. However, in contrast to Anakinra and Rilonacept that block the biological activities of both IL-1 $\beta$ and IL-1 $\alpha$, Canakinumab does not block signaling by IL-1 $\alpha$.

The importance of IL-1 $\beta$ in inflammatory diseases was supported by the results of the Canakinumab Anti-Inflammatory Thrombosis Outcome Study (CANTOS) ${ }^{129}$. This largescale trial tested whether decreasing inflammation with Canakinumab reduced the risk of a second cardiovascular event in patients who previously experienced a heart attack or stroke. The trial demonstrated a benefit of IL-1 $\beta$ inhibition in atherosclerosis. Secondary 
exploratory analysis of a the CANTOS trial also confirmed that IL-1 $\beta$ blockade is of interest in other diseases including gout ${ }^{130-132}$.

Other biologics have been developed to block the IL-1 pathway, however are currently not (yet?) used in clinics. The monoclonal antibody Lutikizumab (ABT-981) has been engineered to target both IL-1 $\alpha$ and IL-1 ${ }^{133}$. Gevokizumab is an IL-1 $\beta$ antagonistic antibody that binds strongly to IL-1 $\beta$ without inhibiting its recruitment to the IL-R. It prevents the engagement of the IL-1 $\beta$-IL-1R signaling complex through its allosteric modulation properties ${ }^{134}$. Furthermore, reagents inhibiting the IL-1R directly have been developed. The AMG108 antibody binds and neutralizes IL-1R, leading to a complete abrogation of IL-1 $\beta$ and IL-1 $\alpha$ signaling ${ }^{135}$.

Inhibition of IL-1 $\beta$ was found to be very effective in typical autoinflammatory diseases such as soJIA ${ }^{136}$. Similarly, CAPS patients with gain of function NLRP3 respond significantly to IL-1 $\beta$ inhibition ${ }^{137}$ indicating that this cytokine is the most relevant product of NLRP3 activation in humans.

In contrast, clinical studies suggest that the efficacy of IL- $1 \beta$ inhibition is modest compared to strategies targeting TNF in RA ${ }^{138}$. This may indicate that beyond inflammasome activation, other inflammatory pathways are predominant in RA. Alternatively, this could support the hypothesis that other products of inflammasome activation contribute to disease pathology. For example, IL-18 or danger signals released during pyroptosis could play a proinflammatory role in RA.

Very few reagents are available to block IL-18 in humans. In a recent clinical trial the use of Tadekinig alfa, a recombinant IL-18 binding protein, showed positive results in adult- 
onset Still's disease ${ }^{139}$, supporting the assumption that IL-18 may contribute to inflammation and immune deregulation in inflammasomopathies.

\section{Caspase-1 inhibitors}

The enzymatic activity of the inflammasome is executed by caspase- 1 proteases. Therefore, these enzymes are attractive targets for pharmacological intervention. Despite two decades

of research no drug based on caspase inhibition has been approved yet ${ }^{140}$. The main challenge is the design of inhibitors that only affect the inflammatory caspases without interfering with proapoptotic caspases. In mice, the inhibition of caspase- 1 was effective in models of rheumatoid arthritis, osteoarthritis and psoriasis ${ }^{140,141}$. Vx-740 and Vx-765, two caspase 1 inhibitors, reached phase II clinical trials for arthritis, epilepsy and psoriasis but were withdrawn, partially due to liver toxicity ${ }^{140}$. New insight into the mechanisms of action of thalidomide, an anti-inflammatory drug that was introduced more than 50 years ago, showed its capacity to block caspase-1 activity ${ }^{142}$. Thalidomide is currently tested in various inflammatory conditions and experimentally used for the treatment of refractory ankylosing spondylitis.

\section{Colchicine}

Colchicine is a widely available, low-cost drug with a range of anti-inflammatory properties that has been used in the form of plant extracts as early as $1550 \mathrm{BC}$ to treat joint swelling ${ }^{143}$. Nowadays, colchicine is commonly used for the treatment of two inflammasomopathies: FMF and gout ${ }^{144}$. Moreover, colchicine anti-inflammatory effects have been shown to have potential benefits in other conditions such as Behcet's disease 
and pericarditis. Moreover, colchicine is considered in prevention or as an add-on therapy to current treatment in a wider spectrum of cardiovascular diseases ${ }^{145}$.

How colchicine inhibits inflammation is still poorly understood. Colchicine inhibits microtubule polymerization, a process that is involved in a variety of cellular processes including maintenance of cellular integrity, signal transduction, protein traffic, and cellular migration. Early studies showed that colchicine treatment of monocytes exposed to goutinducing uric acid crystals prevented the activation of the NLRP3 inflammasome ${ }^{100}$. It was therefore proposed that colchicine may impair microtubule-driven events involved in the assembly of the NLRP3 inflammasome upon exposure to crystals or other activating agonist such as nigericin ${ }^{146}$. In contrast, colchicine has little effect in CAPS patients harboring constitutive activation of NLRP3 suggesting that it may impact inflammasome activity upstream, or in the early steps, of NLRP3 activation ${ }^{147}$.

Colchicine efficacy in FMF has indicated that this compound may also impact the Pyrin inflammasome. In line with this possibility, colchicine was found to block the pyrin inflammasome activation by bacterial toxins and infection with $C$. difficile. In contrast, monocytes from FMF patients with gain-of -function mutations in pyrin were resistant to inflammasome inhibition by colchicine ${ }^{148}$. This may suggest an increased efficacy of colchicine in vivo compared to in vitro experiments. Alternatively, colchicine may modulate an additional mechanism in FMF by targeting a feedback loop or the engagement of other inflammasomes such as NLRP3.

\section{NLRP3 inhibitors}

Increasing evidence for NLRP3 inflammasome involvement in a plethora of inflammatory diseases has led to a sustained enthusiasm by the industry towards the development of 
specific inhibitors. Development of such drugs is facilitated by studies in CAPS patients providing a first and robust testing ground to evaluate the efficacy and specificity of new molecules in blocking NLRP3-mediated inflammation in this NLRP3-driven disease. NLRP3 inhibition is considered for evaluation in rheumatoid arthritis, atherosclerosis, neurodegenerative disorders and obesity-related diseases ${ }^{149}$. Several molecules have been shown to target NLRP3 ${ }^{150}$, and a number of these compounds have promising NLRP3 inhibitory activities.

The compound MCC950 (also known as CRID3) is the NLRP3 inhibitor that has been the most studied to date ${ }^{151,152}$. MCC950 inhibits NLRP3 by directly interacting with the NLRP3 ATPase domain, thereby blocking ATP hydrolysis and NLRP3 oligomerization 151,153. Several studies have shown therapeutic efficacy of MCC950 in a variety of preclinical mouse models, including atherosclerosis, experimental autoimmune encephalomyelitis, diabetes, steatohepatitis and colitis ${ }^{17}$. First, before its identification as a NLRP3 targeting molecule, MCC950 was discovered as an inhibitor of IL-1 $\beta$ activation. This led to the initiation of a clinical trial testing its efficacy in rheumatoid arthritis. Unfortunately, the trial was suspended due to drug toxicity ${ }^{17}$.

Similar to MCC950, a compound called Tranilast was found to bind to the NACHT domain of NLRP3, abolishing its ability to assemble into an inflammasome ${ }^{154}$. This molecule is an analogue of a tryptophan metabolite, that was initially identified as an anti-allergy drug and is presently used in several inflammatory and autoimmune conditions ${ }^{155}$. RA studies in mice have shown that treatment with Tranilast reduced disease in models of collageninduced arthritis ${ }^{156,157}$ and in arthritis induced by adjuvant and streptococcal cell wall products in rats ${ }^{158}$. Its mode of action is still unclear and may not involve inhibition of 
NLRP3 ATPase activity ${ }^{154}$. Tranilast has very low adverse effects, therefore if further studies demonstrate its efficacy and specificity at inhibiting NLRP3, its repositioning in inflammasomopathies could be of great interest and may provide a new therapeutic tool for treating complex diseases with NLRP3 involvement.

The compound CY-09, an analogue of the inhibitor for cystic fibrosis transmembrane conductance regulator channel (CFTR), was recently identified to efficiently inhibit NLRP $3^{159}$. CY-09 specifically binds to the ATPase domain of NLRP3 thereby blocking NLRP3 oligomerization. Preclinical studies showed that CY-09 was efficient at decreasing inflammation in a mouse model of CAPS. Ex-vivo it was found to decrease IL-1 $\beta$ secretion in synovial fluid cells (SFCs) from a patient with gout ${ }^{159}$. Therefore, this molecule could be a candidate for further studies in human arthritic disease.

The Isodon plant, Rabdosia rubescens, and its extracts, were shown in East Asian herbal medicine to have anti-inflammatory properties. Oridonin, a bitter tetracycline diterpenoid compound, was identified as the active compound of these plants extracts ${ }^{160}$. While the mechanism of Oridonin is still unclear, it was shown to inhibit the NLRP3 inflammasome 161. Oridonin decreased the ability of NLRP3 to interact with NEK7, a protein that may modulate its activation ${ }^{161}$.

OLT1177 is a small non-lipophilic molecule that was found to inhibit the NLRP3 inflammasome ${ }^{162}$ and demonstrated suppressed joint inflammation in murine models of acute arthritis ${ }^{163}$. How OLT1177 blocks NLRP3 engagement is still unclear, however the preclinical studies in mice indicate that this molecule could be a promising compound to test in patients with joint disorders. 
Several studies have suggested that certain compounds may function as inflammasome inhibitors including Gyburide ${ }^{164}$, INF39 ${ }^{165}$, BOT-4-one ${ }^{166}, \mathrm{MNS}^{167}$, Bay 11-7082 ${ }^{168}$ and parthenolide ${ }^{168}$. The specificity and efficacy in humans of these compounds need to be further evaluated. However, it is clear that the collection of putative NLRP3 inhibitors is growing fast. The challenge will be to identify the safest and specific compound that will provide the most potent therapeutic benefit to patients.

\section{CONCLUSIONS AND PERSPECTIVES}

The importance of the inflammasome in autoinflammatory diseases has become evident over the past twenty years. In particular genetic studies revealed a contribution of gene variants of inflammasome components to susceptibility for inflammatory diseases including RA, gout and AS (Table 1). While the functionality of some gene polymorphisms were uncovered, further studies are needed to elucidate the role of identified SNPs on inflammasome activity in the context of arthritis. Patients with arthritic diseases have greatly benefited from the development of inflammasome-targeting biologicals and will continue to do so in upcoming years. Research into the mechanisms of different inflammasome activation has spiked, which will fuel the possibility for designing highly specific and efficient inhibitors. With the recent discovery of GSDMD as a major driver of pyroptosis, small molecule inhibitors that block GSDMD function are expected to emerge for possible treatment of inflammatory conditions including arthritis. Moreover, clinical efficacy of the diverse selection of NLRP3 inhibitors under development will come from trials which are typically performed in CAPS patients. While CAPS is a rare disease, these inhibitors could become blockbuster drugs provided that their efficacy will be 
confirmed in more common inflammatory diseases. To facilitate efficient transition of newly discovered inhibitors to (pre)-clinical studies, using human cellular models over animal models is imperative. Differences between human and mouse inflammasome biology, such as the lack of CARD8 in mice, impacts conclusions about treatment efficacies obtained with murine disease models. Through design of relevant cellular models incorporating patient mutations or direct use of patient-derived cells we may identify clinically beneficial treatments faster and with more certainty.

\section{ACKOWLEDGEMENTS}

FM is supported by grants from the Swiss National Science Foundation (310030_173152) and the Institute of Rheumatology Research (IRR, https://www.irr-research.org). LS is supported by research programme ZonMW with project number 452183005 , which is (partly) financed by the Dutch Research Council (NWO). 


\section{Figure legends:}

Figure 1. Schematic overview of inflammasome platforms. The structure and domain interactions of Nod-like receptor (NLR) inflammasomes (A) and non-NLR inflammasomes (B) are depicted. NLRP3, Pyrin and AIM2 all contain a N-terminal recruitment domain PYD whereas NLRC4 contains a CARD domain at its N-terminus. PYD-PYD interactions occur with the adaptor protein ASC which in turn interacts with Caspase-1 through CARD-CARD interactions. The N-terminal CARD domain of NLRC4 directly interacts with Caspase-1. NLRP1 harbors its CARD recruitment domain at the Cterminus which also directly interacts with Caspase-1.

Figure 2. Local and systemic effects of inflammasome activation. Innate immune cells such as monocytes and neutrophils receive priming signals (signal 1) that induce NFkB activation. NFאB upregulates gene transcription of inflammasome components NLRP3, Caspase-1 and ASC, as well as inflammasome substrates proIL1- $\beta$, proIL-18 and GSDMD. Upon delivery of inflammasome activation signals (signal 2) assembly of the inflammasome complex occurs which results in activation of the caspasel enzyme now able to cleave its substrates. Cleaved GSDMD forms membrane pores through which mature cytokines IL-1 $\beta$ and IL-18 are secreted. IL-1 $\beta$ and IL-18 bind to their receptors IL1R and IL-18R, respectively. IL-1R/IL-18R signalling drives various local and systemic reactions including a positive inflammasome feedback loop by providing signal 1, stimulation of many different target cells that promote activating a local inflammatory 
response, and initiation of systemic inflammatory functions such as neutrophil recruitment, fever and production of acute phase proteins.

Figure 3. Inhibitors targeting inflammasome activity. The molecular targets of selected anti-inflammatory drugs are depicted. Inhibitors for which inhibition of inflammasome was observed, however the molecular target is unknown are listed directly beneath the inflammasome complex. Arthritic diseases are commonly treated with Anakinra, Rilonacept, Canakinumab, Thalidomide or Colchicine depending on the disease type. 


\section{REFERENCES}

1. Martinon F, Burns K, Tschopp J. The inflammasome: a molecular platform triggering activation of inflammatory caspases and processing of proIL-beta. $\mathrm{Mol}$ Cell. 2002;10(2):417-426.

2. Dinarello CA. The IL-1 family of cytokines and receptors in rheumatic diseases. Nat Rev Rheumatol. 2019;15(10):612-632.

3. Broz P, Dixit VM. Inflammasomes: mechanism of assembly, regulation and signalling. Nat Rev Immunol. 2016;16(7):407-420.

4. Harapas CR, Steiner A, Davidson S, Masters SL. An Update on Autoinflammatory Diseases: Inflammasomopathies. Curr Rheumatol Rep. 2018;20(7):40.

5. Latz E, Duewell P. NLRP3 inflammasome activation in inflammaging. Semin Immunol. 2018;40:61-73.

6. Shin J, Lee KH, Joo YH, et al. Inflammasomes and autoimmune and rheumatic diseases: A comprehensive review. J Autoimmun. 2019;103:102299.

7. Martinon F, Tschopp J. NLRs join TLRs as innate sensors of pathogens. Trends Immunol. 2005;26(8):447-454.

8. Burdett H, Bentham AR, Williams SJ, et al. The Plant "Resistosome": Structural Insights into Immune Signaling. Cell Host Microbe. 2019;26(2):193-201.

9. Wang J, Hu M, Wang J, et al. Reconstitution and structure of a plant NLR resistosome conferring immunity. Science. 2019;364(6435).

10. Urbach JM, Ausubel FM. The NBS-LRR architectures of plant R-proteins and metazoan NLRs evolved in independent events. Proc Natl Acad Sci U S A. 2017;114(5):1063-1068.

11. Leipe DD, Koonin EV, Aravind L. STAND, a class of P-loop NTPases including animal and plant regulators of programmed cell death: multiple, complex domain architectures, unusual phyletic patterns, and evolution by horizontal gene transfer. J Mol Biol. 2004;343(1):1-28.

12. MacDonald JA, Wijekoon CP, Liao KC, Muruve DA. Biochemical and structural aspects of the ATP-binding domain in inflammasome-forming human NLRP proteins. IUBMB Life. 2013;65(10):851-862.

13. Duncan JA, Canna SW. The NLRC4 Inflammasome. Immunol Rev. 2018;281(1):115-123.

14. Petrilli V, Papin S, Dostert C, Mayor A, Martinon F, Tschopp J. Activation of the NALP3 inflammasome is triggered by low intracellular potassium concentration. Cell Death Differ. 2007;14(9):1583-1589. 
15. Karki P, Seong C, Kim JE, et al. Intracellular K(+) inhibits apoptosis by suppressing the Apaf-1 apoptosome formation and subsequent downstream pathways but not cytochrome c release. Cell Death Differ. 2007;14(12):20682075.

16. Chen J, Chen ZJ. PtdIns4P on dispersed trans-Golgi network mediates NLRP3 inflammasome activation. Nature. 2018;564(7734):71-76.

17. Swanson KV, Deng M, Ting JP. The NLRP3 inflammasome: molecular activation and regulation to therapeutics. Nat Rev Immunol. 2019;19(8):477-489.

18. Hoffman HM, Mueller JL, Broide DH, Wanderer AA, Kolodner RD. Mutation of a new gene encoding a putative pyrin-like protein causes familial cold autoinflammatory syndrome and Muckle-Wells syndrome. Nat Genet. 2001;29(3):301-305.

19. Booshehri LM, Hoffman HM. CAPS and NLRP3. J Clin Immunol. 2019;39(3):277-286.

20. Agostini L, Martinon F, Burns K, McDermott MF, Hawkins PN, Tschopp J. NALP3 forms an IL-1beta-processing inflammasome with increased activity in Muckle-Wells autoinflammatory disorder. Immunity. 2004;20(3):319-325.

21. Boyden ED, Dietrich WF. Nalp1b controls mouse macrophage susceptibility to anthrax lethal toxin. Nat Genet. 2006;38(2):240-244.

22. Okondo MC, Rao SD, Taabazuing CY, et al. Inhibition of Dpp8/9 Activates the Nlrp1b Inflammasome. Cell Chem Biol. 2018;25(3):262-267 e265.

23. Mitchell PS, Sandstrom A, Vance RE. The NLRP1 inflammasome: new mechanistic insights and unresolved mysteries. Curr Opin Immunol. 2019;60:3745.

24. Zhong FL, Mamai O, Sborgi L, et al. Germline NLRP1 Mutations Cause Skin Inflammatory and Cancer Susceptibility Syndromes via Inflammasome Activation. Cell. 2016;167(1):187-202 e117.

25. Grandemange S, Sanchez E, Louis-Plence P, et al. A new autoinflammatory and autoimmune syndrome associated with NLRP1 mutations: NAIAD (NLRP1associated autoinflammation with arthritis and dyskeratosis). Ann Rheum Dis. 2017;76(7):1191-1198.

26. Jin Y, Mailloux CM, Gowan $\mathrm{K}$, et al. NALP1 in vitiligo-associated multiple autoimmune disease. N Engl J Med. 2007;356(12):1216-1225.

27. Yu CH, Moecking J, Geyer M, Masters SL. Mechanisms of NLRP1-Mediated Autoinflammatory Disease in Humans and Mice. J Mol Biol. 2018;430(2):142152.

28. Pathan N, Marusawa H, Krajewska M, et al. TUCAN, an antiapoptotic caspaseassociated recruitment domain family protein overexpressed in cancer. $J$ Biol Chem. 2001;276(34):32220-32229. 
29. Bagnall RD, Roberts RG, Mirza MM, Torigoe T, Prescott NJ, Mathew CG. Novel isoforms of the CARD8 (TUCAN) gene evade a nonsense mutation. Eur J Hum Genet. 2008;16(5):619-625.

30. Johnson DC, Taabazuing CY, Okondo MC, et al. DPP8/DPP9 inhibitor-induced pyroptosis for treatment of acute myeloid leukemia. Nat Med. 2018;24(8):11511156.

31. Ito S, Hara Y, Kubota T. CARD8 is a negative regulator for NLRP3 inflammasome, but mutant NLRP3 in cryopyrin-associated periodic syndromes escapes the restriction. Arthritis Res Ther. 2014;16(1):R52.

32. Fontalba A, Martinez-Taboada V, Gutierrez O, et al. Deficiency of the NFkappaB inhibitor caspase activating and recruitment domain 8 in patients with rheumatoid arthritis is associated with disease severity. J Immunol. 2007;179(7):4867-4873.

33. Kastbom A, Verma D, Eriksson P, Skogh T, Wingren G, Soderkvist P. Genetic variation in proteins of the cryopyrin inflammasome influences susceptibility and severity of rheumatoid arthritis (the Swedish TIRA project). Rheumatology (Oxford). 2008;47(4):415-417.

34. Mathews RJ, Robinson JI, Battellino M, et al. Evidence of NLRP3-inflammasome activation in rheumatoid arthritis (RA); genetic variants within the NLRP3inflammasome complex in relation to susceptibility to RA and response to antiTNF treatment. Ann Rheum Dis. 2014;73(6):1202-1210.

35. Yang CA, Huang ST, Chiang BL. Association of NLRP3 and CARD8 genetic polymorphisms with juvenile idiopathic arthritis in a Taiwanese population. Scand J Rheumatol. 2014;43(2):146-152.

36. Lee SW, Lee SS, Oh DH, et al. Genetic Association for P2X7R rs3751142 and CARD8 rs2043211 Polymorphisms for Susceptibility of Gout in Korean Men: Multi-Center Study. J Korean Med Sci. 2016;31(10):1566-1570.

37. Ancient missense mutations in a new member of the RoRet gene family are likely to cause familial Mediterranean fever. The International FMF Consortium. Cell. 1997;90(4):797-807.

38. French FMFC. A candidate gene for familial Mediterranean fever. Nat Genet. 1997;17(1):25-31.

39. Yang $\mathrm{J}, \mathrm{Xu} \mathrm{H}$, Shao F. Immunological function of familial Mediterranean fever disease protein Pyrin. Sci China Life Sci. 2014;57(12):1156-1161.

40. Canete JD, Arostegui JI, Queiro R, et al. An unexpectedly high frequency of MEFV mutations in patients with anti-citrullinated protein antibody-negative palindromic rheumatism. Arthritis Rheum. 2007;56(8):2784-2788.

41. Ayaz NA, Ozen S, Bilginer Y, et al. MEFV mutations in systemic onset juvenile idiopathic arthritis. Rheumatology (Oxford). 2009;48(1):23-25.

42. Lugrin J, Martinon F. The AIM2 inflammasome: Sensor of pathogens and cellular perturbations. Immunol Rev. 2018;281(1):99-114. 
43. Baum R, Sharma S, Carpenter S, et al. Cutting edge: AIM2 and endosomal TLRs differentially regulate arthritis and autoantibody production in DNase II-deficient mice. J Immunol. 2015;194(3):873-877.

44. Jakobs C, Perner S, Hornung V. AIM2 Drives Joint Inflammation in a Self-DNA Triggered Model of Chronic Polyarthritis. PLoS One. 2015;10(6):e0131702.

45. Hawkins PN, Lachmann HJ, McDermott MF. Interleukin-1-receptor antagonist in the Muckle-Wells syndrome. N Engl J Med. 2003;348(25):2583-2584.

46. Orning P, Lien E, Fitzgerald KA. Gasdermins and their role in immunity and inflammation. J Exp Med. 2019.

47. Chen KW, Gross CJ, Sotomayor FV, et al. The neutrophil NLRC4 inflammasome selectively promotes IL-1beta maturation without pyroptosis during acute Salmonella challenge. Cell Rep. 2014;8(2):570-582.

48. Gaidt MM, Ebert TS, Chauhan D, et al. Human Monocytes Engage an Alternative Inflammasome Pathway. Immunity. 2016;44(4):833-846.

49. Kayagaki N, Stowe IB, Lee BL, et al. Caspase-11 cleaves gasdermin D for noncanonical inflammasome signalling. Nature. 2015;526(7575):666-671.

50. Shi J, Zhao Y, Wang K, et al. Cleavage of GSDMD by inflammatory caspases determines pyroptotic cell death. Nature. 2015;526(7575):660-665.

51. Evavold CL, Ruan J, Tan Y, Xia S, Wu H, Kagan JC. The Pore-Forming Protein Gasdermin D Regulates Interleukin-1 Secretion from Living Macrophages. Immunity. 2018;48(1):35-44 e36.

52. Heilig R, Dick MS, Sborgi L, Meunier E, Hiller S, Broz P. The Gasdermin-D pore acts as a conduit for IL-1beta secretion in mice. Eur J Immunol. 2018;48(4):584592.

53. Ruhl S, Shkarina K, Demarco B, Heilig R, Santos JC, Broz P. ESCRT-dependent membrane repair negatively regulates pyroptosis downstream of GSDMD activation. Science. 2018;362(6417):956-960.

54. Medzhitov R. Origin and physiological roles of inflammation. Nature. 2008;454(7203):428-435.

55. Erlich Z, Shlomovitz I, Edry-Botzer L, et al. Macrophages, rather than DCs, are responsible for inflammasome activity in the GM-CSF BMDC model. Nat Immunol. 2019;20(4):397-406.

56. Man SM. Inflammasomes in the gastrointestinal tract: infection, cancer and gut microbiota homeostasis. Nat Rev Gastroenterol Hepatol. 2018;15(12):721-737.

57. Monroe KM, Yang Z, Johnson JR, et al. IFI16 DNA sensor is required for death of lymphoid CD4 T cells abortively infected with HIV. Science. 2014;343(6169):428-432.

58. Arbore G, West EE, Spolski R, et al. T helper 1 immunity requires complementdriven NLRP3 inflammasome activity in CD4(+) T cells. Science. 2016;352(6292):aad1210. 
59. Martin BN, Wang C, Zhang CJ, et al. T cell-intrinsic ASC critically promotes T(H)17-mediated experimental autoimmune encephalomyelitis. Nat Immunol. 2016;17(5):583-592.

60. Helmick CG, Felson DT, Lawrence RC, et al. Estimates of the prevalence of arthritis and other rheumatic conditions in the United States. Part I. Arthritis Rheum. 2008;58(1):15-25.

61. Scott DL, Symmons DP, Coulton BL, Popert AJ. Long-term outcome of treating rheumatoid arthritis: results after 20 years. Lancet. 1987;1(8542):1108-1111.

62. Scott DL, Wolfe F, Huizinga TW. Rheumatoid arthritis. Lancet. 2010;376(9746):1094-1108.

63. Burska A, Boissinot M, Ponchel F. Cytokines as biomarkers in rheumatoid arthritis. Mediators Inflamm. 2014;2014:545493.

64. Ippagunta SK, Brand DD, Luo J, et al. Inflammasome-independent role of apoptosis-associated speck-like protein containing a CARD (ASC) in T cell priming is critical for collagen-induced arthritis. J Biol Chem. 2010;285(16):12454-12462.

65. Kolly L, Karababa M, Joosten LA, et al. Inflammatory role of ASC in antigeninduced arthritis is independent of caspase-1, NALP-3, and IPAF. J Immunol. 2009; 183(6):4003-4012.

66. Zhang Y, Zheng Y, Li H. NLRP3 Inflammasome Plays an Important Role in the Pathogenesis of Collagen-Induced Arthritis. Mediators Inflamm. 2016;2016:9656270.

67. Shin TH, Kim HS, Kang TW, et al. Human umbilical cord blood-stem cells direct macrophage polarization and block inflammasome activation to alleviate rheumatoid arthritis. Cell Death Dis. 2016;7(12):e2524.

68. Li XF, Shen WW, Sun YY, et al. MicroRNA-20a negatively regulates expression of NLRP3-inflammasome by targeting TXNIP in adjuvant-induced arthritis fibroblast-like synoviocytes. Joint Bone Spine. 2016;83(6):695-700.

69. Li Y, Zheng JY, Liu JQ, et al. Succinate/NLRP3 Inflammasome Induces Synovial Fibroblast Activation: Therapeutical Effects of Clematichinenoside AR on Arthritis. Front Immunol. 2016;7:532.

70. Vande Walle L, Van Opdenbosch N, Jacques P, et al. Negative regulation of the NLRP3 inflammasome by A20 protects against arthritis. Nature. 2014;512(7512):69-73.

71. Joosten LA, Abdollahi-Roodsaz S, Heuvelmans-Jacobs M, et al. T cell dependence of chronic destructive murine arthritis induced by repeated local activation of Toll-like receptor-driven pathways: crucial role of both interleukin1beta and interleukin-17. Arthritis Rheum. 2008;58(1):98-108.

72. Joosten LA, Netea MG, Fantuzzi G, et al. Inflammatory arthritis in caspase 1 gene-deficient mice: contribution of proteinase 3 to caspase 1-independent 
production of bioactive interleukin-1beta. Arthritis Rheum. 2009;60(12):36513662.

73. Ji H, Pettit A, Ohmura K, et al. Critical roles for interleukin 1 and tumor necrosis factor alpha in antibody-induced arthritis. $J$ Exp Med. 2002;196(1):77-85.

74. Choulaki C, Papadaki G, Repa A, et al. Enhanced activity of NLRP3 inflammasome in peripheral blood cells of patients with active rheumatoid arthritis. Arthritis Res Ther. 2015;17:257.

75. Addobbati C, da Cruz HLA, Adelino JE, et al. Polymorphisms and expression of inflammasome genes are associated with the development and severity of rheumatoid arthritis in Brazilian patients. Inflamm Res. 2018;67(3):255-264.

76. Xie Q, Wei M, Zhang B, et al. MicroRNA33 regulates the NLRP3 inflammasome signaling pathway in macrophages. Mol Med Rep. 2018;17(2):3318-3327.

77. Ruscitti P, Cipriani P, Di Benedetto P, et al. Monocytes from patients with rheumatoid arthritis and type 2 diabetes mellitus display an increased production of interleukin (IL)-1beta via the nucleotide-binding domain and leucine-rich repeat containing family pyrin 3(NLRP3)-inflammasome activation: a possible implication for therapeutic decision in these patients. Clin Exp Immunol. 2015;182(1):35-44.

78. Jenko B, Praprotnik S, Tomsic M, Dolzan V. NLRP3 and CARD8 Polymorphisms Influence Higher Disease Activity in Rheumatoid Arthritis. $J$ Med Biochem. 2016;35(3):319-323.

79. Rosengren S, Hoffman HM, Bugbee W, Boyle DL. Expression and regulation of cryopyrin and related proteins in rheumatoid arthritis synovium. Ann Rheum Dis. 2005;64(5):708-714.

80. Kolly L, Busso N, Palmer G, Talabot-Ayer D, Chobaz V, So A. Expression and function of the NALP3 inflammasome in rheumatoid synovium. Immunology. 2010;129(2):178-185.

81. Joosten LA, Radstake TR, Lubberts E, et al. Association of interleukin-18 expression with enhanced levels of both interleukin-1beta and tumor necrosis factor alpha in knee synovial tissue of patients with rheumatoid arthritis. Arthritis Rheum. 2003;48(2):339-347.

82. Gouda EA, Aboulata AA, Elharoun AS, et al. Interleukin-18 expression in rheumatoid artheritis synovial tissue and its relation to disease activity. Egypt $J$ Immunol. 2007;14(2):1-10.

83. Cai LP, Zhou LJ, Lu SY, et al. Association of IL-18 promoter gene polymorphisms with rheumatoid arthritis: a meta-analysis. Mol Biol Rep. 2014;41(12):8211-8217.

84. Ye XJ, Tang B, Ma Z, Kang AH, Myers LK, Cremer MA. The roles of interleukin-18 in collagen-induced arthritis in the BB rat. Clin Exp Immunol. 2004;136(3):440-447. 
85. Ruth JH, Park CC, Amin MA, et al. Interleukin-18 as an in vivo mediator of monocyte recruitment in rodent models of rheumatoid arthritis. Arthritis Res Ther. 2010;12(3):R118.

86. Amin MA, Rabquer BJ, Mansfield PJ, et al. Interleukin 18 induces angiogenesis in vitro and in vivo via Src and Jnk kinases. Ann Rheum Dis. 2010;69(12):22042212.

87. Zhang W, Cong XL, Qin YH, He ZW, He DY, Dai SM. IL-18 upregulates the production of key regulators of osteoclastogenesis from fibroblast-like synoviocytes in rheumatoid arthritis. Inflammation. 2013;36(1):103-109.

88. Sui J, Li H, Fang Y, et al. NLRP1 gene polymorphism influences gene transcription and is a risk factor for rheumatoid arthritis in han chinese. Arthritis Rheum. 2012;64(3):647-654.

89. Zhu L, Li J, Guo L, et al. Activation of NALP1 inflammasomes in rats with adjuvant arthritis; a novel therapeutic target of carboxyamidotriazole in a model of rheumatoid arthritis. Br J Pharmacol. 2015;172(13):3446-3459.

90. Zhang L, Dong Y, Zou F, Wu M, Fan C, Ding Y. 11 beta-Hydroxysteroid dehydrogenase 1 inhibition attenuates collagen-induced arthritis. Int Immunopharmacol. 2013;17(3):489-494.

91. Li F, Guo N, Ma Y, Ning B, Wang Y, Kou L. Inhibition of P2X4 suppresses joint inflammation and damage in collagen-induced arthritis. Inflammation. 2014;37(1):146-153.

92. Zhang QB, Qing YF, He YL, Xie WG, Zhou JG. Association of NLRP3 polymorphisms with susceptibility to primary gouty arthritis in a Chinese Han population. Clin Rheumatol. 2018;37(1):235-244.

93. McKinney C, Stamp LK, Dalbeth N, et al. Multiplicative interaction of functional inflammasome genetic variants in determining the risk of gout. Arthritis Res Ther. 2015; 17:288.

94. Chang WC, Jan Wu YJ, Chung WH, et al. Genetic variants of PPAR-gamma coactivator 1B augment NLRP3-mediated inflammation in gouty arthritis. Rheumatology (Oxford). 2017;56(3):457-466.

95. Choe JY, Kim SK. Clinical significance of serum NLRP3 levels in patients with chronic gouty arthritis. Joint Bone Spine. 2018;85(2):257-258.

96. Cavalcanti NG, Marques CD, Lins ELTU, et al. Cytokine Profile in Gout: Inflammation Driven by IL-6 and IL-18? Immunol Invest. 2016;45(5):383-395.

97. Son CN, Bang SY, Kim JH, Choi CB, Kim TH, Jun JB. Caspase-1 level in synovial fluid is high in patients with spondyloarthropathy but not in patients with gout. J Korean Med Sci. 2013;28(9):1289-1292.

98. Busso N, So A. Mechanisms of inflammation in gout. Arthritis Res Ther. 2010;12(2):206. 
99. Amaral FA, Costa VV, Tavares LD, et al. NLRP3 inflammasome-mediated neutrophil recruitment and hypernociception depend on leukotriene $\mathrm{B}(4)$ in a murine model of gout. Arthritis Rheum. 2012;64(2):474-484.

100. Martinon F, Petrilli V, Mayor A, Tardivel A, Tschopp J. Gout-associated uric acid crystals activate the NALP3 inflammasome. Nature. 2006;440(7081):237-241.

101. Hoffman HM, Scott P, Mueller JL, et al. Role of the leucine-rich repeat domain of cryopyrin/NALP3 in monosodium urate crystal-induced inflammation in mice. Arthritis Rheum. 2010;62(7):2170-2179.

102. Cumpelik A, Ankli B, Zecher D, Schifferli JA. Neutrophil microvesicles resolve gout by inhibiting C5a-mediated priming of the inflammasome. Ann Rheum Dis. 2016;75(6):1236-1245.

103. Khameneh HJ, Ho AW, Laudisi F, et al. C5a Regulates IL-1beta Production and Leukocyte Recruitment in a Murine Model of Monosodium Urate CrystalInduced Peritonitis. Front Pharmacol. 2017;8:10.

104. Joosten LA, Netea MG, Mylona E, et al. Engagement of fatty acids with Toll-like receptor 2 drives interleukin-1beta production via the ASC/caspase 1 pathway in monosodium urate monohydrate crystal-induced gouty arthritis. Arthritis Rheum. 2010;62(11):3237-3248.

105. Mylona EE, Mouktaroudi M, Crisan TO, et al. Enhanced interleukin-1beta production of PBMCs from patients with gout after stimulation with Toll-like receptor-2 ligands and urate crystals. Arthritis Res Ther. 2012;14(4):R158.

106. Migita K, Koga T, Satomura K, et al. Serum amyloid A triggers the mosodium urate -mediated mature interleukin-1beta production from human synovial fibroblasts. Arthritis Res Ther. 2012;14(3):R119.

107. Riteau N, Baron L, Villeret B, et al. ATP release and purinergic signaling: a common pathway for particle-mediated inflammasome activation. Cell Death Dis. 2012;3:e403.

108. Yokose K, Sato S, Asano T, et al. TNF-alpha potentiates uric acid-induced interleukin-1beta (IL-1beta) secretion in human neutrophils. Mod Rheumatol. 2018;28(3):513-517.

109. Xiao J, Zhang XL, Fu C, et al. Soluble uric acid increases NALP3 inflammasome and interleukin-1beta expression in human primary renal proximal tubule epithelial cells through the Toll-like receptor 4-mediated pathway. Int J Mol Med. 2015;35(5):1347-1354.

110. Alberts BM, Barber JS, Sacre SM, Davies KA, Ghezzi P, Mullen LM. Precipitation of Soluble Uric Acid Is Necessary for In Vitro Activation of the NLRP3 Inflammasome in Primary Human Monocytes. J Rheumatol. 2019.

111. Brown MA, Kenna T, Wordsworth BP. Genetics of ankylosing spondylitis-insights into pathogenesis. Nat Rev Rheumatol. 2016;12(2):81-91. 
112. Ranganathan V, Gracey E, Brown MA, Inman RD, Haroon N. Pathogenesis of ankylosing spondylitis - recent advances and future directions. Nat Rev Rheumatol. 2017;13(6):359-367.

113. Maksymowych WP, Rahman P, Reeve JP, Gladman DD, Peddle L, Inman RD. Association of the IL1 gene cluster with susceptibility to ankylosing spondylitis: an analysis of three Canadian populations. Arthritis Rheum. 2006;54(3):974-985.

114. Timms AE, Crane AM, Sims AM, et al. The interleukin 1 gene cluster contains a major susceptibility locus for ankylosing spondylitis. Am J Hum Genet. 2004;75(4):587-595.

115. van der Paardt M, Crusius JB, Garcia-Gonzalez MA, et al. Interleukin-1beta and interleukin-1 receptor antagonist gene polymorphisms in ankylosing spondylitis. Rheumatology (Oxford). 2002;41(12):1419-1423.

116. Sims AM, Timms AE, Bruges-Armas J, et al. Prospective meta-analysis of interleukin 1 gene complex polymorphisms confirms associations with ankylosing spondylitis. Ann Rheum Dis. 2008;67(9):1305-1309.

117. Monnet D, Kadi A, Izac B, et al. Association between the IL-1 family gene cluster and spondyloarthritis. Ann Rheum Dis. 2012;71(6):885-890.

118. Vazquez-Del Mercado M, Garcia-Gonzalez A, Munoz-Valle JF, et al. Interleukin 1 beta (IL-1beta), IL-10, tumor necrosis factor-alpha, and cellular proliferation index in peripheral blood mononuclear cells in patients with ankylosing spondylitis. J Rheumatol. 2002;29(3):522-526.

119. Kim SK, Cho YJ, Choe JY. NLRP3 inflammasomes and NLRP3 inflammasomederived proinflammatory cytokines in peripheral blood mononuclear cells of patients with ankylosing spondylitis. Clin Chim Acta. 2018;486:269-274.

120. Kastbom A, Klingberg E, Verma D, et al. Genetic variants in CARD8 but not in NLRP3 are associated with ankylosing spondylitis. Scand J Rheumatol. 2013;42(6):465-468.

121. Ziaee V, Maddah M, Harsini S, et al. Association of interleukin-1 family gene polymorphisms with juvenile idiopathic arthritis in Iranian population. Allergol Immunopathol (Madr). 2016;44(6):542-546.

122. de Jager W, Hoppenreijs EP, Wulffraat NM, Wedderburn LR, Kuis W, Prakken BJ. Blood and synovial fluid cytokine signatures in patients with juvenile idiopathic arthritis: a cross-sectional study. Ann Rheum Dis. 2007;66(5):589-598.

123. Sawhney S, Woo P, Murray KJ. Macrophage activation syndrome: a potentially fatal complication of rheumatic disorders. Arch Dis Child. 2001;85(5):421-426.

124. Pascual V, Allantaz F, Arce E, Punaro M, Banchereau J. Role of interleukin-1 (IL-1) in the pathogenesis of systemic onset juvenile idiopathic arthritis and clinical response to IL-1 blockade. J Exp Med. 2005;201(9):1479-1486.

125. Lotito AP, Campa A, Silva CA, Kiss MH, Mello SB. Interleukin 18 as a marker of disease activity and severity in patients with juvenile idiopathic arthritis. $J$ Rheumatol. 2007;34(4):823-830. 
126. Shimizu M, Nakagishi Y, Yachie A. Distinct subsets of patients with systemic juvenile idiopathic arthritis based on their cytokine profiles. Cytokine. 2013;61(2):345-348.

127. Bousfiha A, Jeddane L, Picard C, et al. The 2017 IUIS Phenotypic Classification for Primary Immunodeficiencies. J Clin Immunol. 2018;38(1):129-143.

128. Economides AN, Carpenter LR, Rudge JS, et al. Cytokine traps: multicomponent, high-affinity blockers of cytokine action. Nat Med. 2003;9(1):47-52.

129. Ridker PM, Everett BM, Thuren T, et al. Antiinflammatory Therapy with Canakinumab for Atherosclerotic Disease. N Engl J Med. 2017;377(12):11191131.

130. Schlesinger N, Alten RE, Bardin T, et al. Canakinumab for acute gouty arthritis in patients with limited treatment options: results from two randomised, multicentre, active-controlled, double-blind trials and their initial extensions. Ann Rheum Dis. 2012;71(11):1839-1848.

131. Solomon DH, Glynn RJ, MacFadyen JG, et al. Relationship of Interleukin-1beta Blockade With Incident Gout and Serum Uric Acid Levels: Exploratory Analysis of a Randomized Controlled Trial. Ann Intern Med. 2018;169(8):535-542.

132. Lyseng-Williamson KA. Canakinumab: a guide to its use in acute gouty arthritis flares. BioDrugs. 2013;27(4):401-406.

133. Fleischmann RM, Bliddal H, Blanco FJ, et al. A Phase II Trial of Lutikizumab, an Anti-Interleukin-1alpha/beta Dual Variable Domain Immunoglobulin, in Knee Osteoarthritis Patients With Synovitis. Arthritis Rheumatol. 2019;71(7):10561069.

134. Geiler J, McDermott MF. Gevokizumab, an anti-IL-1beta mAb for the potential treatment of type 1 and 2 diabetes, rheumatoid arthritis and cardiovascular disease. Curr Opin Mol Ther. 2010;12(6):755-769.

135. Cardiel MH, Tak PP, Bensen $\mathrm{W}$, et al. A phase 2 randomized, double-blind study of AMG 108, a fully human monoclonal antibody to IL-1R, in patients with rheumatoid arthritis. Arthritis Res Ther. 2010;12(5):R192.

136. Ruperto N, Brunner HI, Quartier P, et al. Two randomized trials of canakinumab in systemic juvenile idiopathic arthritis. N Engl J Med. 2012;367(25):2396-2406.

137. Jesus AA, Goldbach-Mansky R. IL-1 blockade in autoinflammatory syndromes. Annu Rev Med. 2014;65:223-244.

138. Genovese MC, Cohen S, Moreland L, et al. Combination therapy with etanercept and anakinra in the treatment of patients with rheumatoid arthritis who have been treated unsuccessfully with methotrexate. Arthritis Rheum. 2004;50(5):14121419.

139. Kiltz U, Kiefer D, Braun J, Schiffrin EJ, Girard-Guyonvarc'h C, Gabay C. Prolonged treatment with Tadekinig alfa in adult-onset Still's disease. Ann Rheum Dis. 2018. 
140. Kudelova J, Fleischmannova J, Adamova E, Matalova E. Pharmacological caspase inhibitors: research towards therapeutic perspectives. J Physiol Pharmacol. 2015;66(4):473-482.

141. Rudolphi K, Gerwin N, Verzijl N, van der Kraan P, van den Berg W. Pralnacasan, an inhibitor of interleukin-1beta converting enzyme, reduces joint damage in two murine models of osteoarthritis. Osteoarthritis Cartilage. 2003;11(10):738-746.

142. Keller M, Sollberger G, Beer HD. Thalidomide inhibits activation of caspase-1. $J$ Immunol. 2009;183(9):5593-5599.

143. Slobodnick A, Shah B, Krasnokutsky S, Pillinger MH. Update on colchicine, 2017. Rheumatology (Oxford). 2018;57(suppl_1):i4-i11.

144. Leung YY, Yao Hui LL, Kraus VB. Colchicine--Update on mechanisms of action and therapeutic uses. Semin Arthritis Rheum. 2015;45(3):341-350.

145. Nidorf SM, Thompson PL. Why Colchicine Should Be Considered for Secondary Prevention of Atherosclerosis: An Overview. Clin Ther. 2019;41(1):41-48.

146. Misawa T, Takahama M, Kozaki T, et al. Microtubule-driven spatial arrangement of mitochondria promotes activation of the NLRP3 inflammasome. Nat Immunol. 2013;14(5):454-460.

147. Hoss F, Latz E. Inhibitory effects of colchicine on inflammasomes. Atherosclerosis. 2018;273:153-154.

148. Van Gorp H, Saavedra PH, de Vasconcelos NM, et al. Familial Mediterranean fever mutations lift the obligatory requirement for microtubules in Pyrin inflammasome activation. Proc Natl Acad Sci U S A. 2016;113(50):14384-14389.

149. Mullard A. NLRP3 inhibitors stoke anti-inflammatory ambitions. Nat Rev Drug Discov. 2019;18(6):405-407.

150. Yang Y, Wang H, Kouadir M, Song H, Shi F. Recent advances in the mechanisms of NLRP3 inflammasome activation and its inhibitors. Cell Death Dis. 2019;10(2):128.

151. Coll RC, Hill JR, Day CJ, et al. MCC950 directly targets the NLRP3 ATPhydrolysis motif for inflammasome inhibition. Nat Chem Biol. 2019;15(6):556559.

152. Perregaux DG, McNiff P, Laliberte R, et al. Identification and characterization of a novel class of interleukin-1 post-translational processing inhibitors. $J$ Pharmacol Exp Ther. 2001;299(1):187-197.

153. Tapia-Abellan A, Angosto-Bazarra D, Martinez-Banaclocha H, et al. MCC950 closes the active conformation of NLRP3 to an inactive state. Nat Chem Biol. 2019;15(6):560-564.

154. Huang $\mathrm{Y}$, Jiang $\mathrm{H}$, Chen $\mathrm{Y}$, et al. Tranilast directly targets NLRP3 to treat inflammasome-driven diseases. EMBO Mol Med. 2018;10(4).

155. Darakhshan S, Pour AB. Tranilast: a review of its therapeutic applications. Pharmacol Res. 2015;91:15-28. 
156. Inglis JJ, Criado G, Andrews M, Feldmann M, Williams RO, Selley ML. The anti-allergic drug, N-(3',4'-dimethoxycinnamonyl) anthranilic acid, exhibits potent anti-inflammatory and analgesic properties in arthritis. Rheumatology (Oxford). 2007;46(9):1428-1432.

157. Shiota N, Kovanen PT, Eklund KK, et al. The anti-allergic compound tranilast attenuates inflammation and inhibits bone destruction in collagen-induced arthritis in mice. Br J Pharmacol. 2010;159(3):626-635.

158. Nagate T, Tamura T, Sato F, Kuroda J, Nakayama J, Shibata N. Tranilast suppresses the disease development of the adjuvant- and streptococcal cell wallinduced arthritis in rats. J Pharmacol Sci. 2007;105(1):48-56.

159. Jiang $\mathrm{H}, \mathrm{He} \mathrm{H}$, Chen $\mathrm{Y}$, et al. Identification of a selective and direct NLRP3 inhibitor to treat inflammatory disorders. J Exp Med. 2017;214(11):3219-3238.

160. Xu J, Wold EA, Ding Y, Shen Q, Zhou J. Therapeutic Potential of Oridonin and Its Analogs: From Anticancer and Antiinflammation to Neuroprotection. Molecules. 2018;23(2).

161. He HB, Jiang H, Chen Y, et al. Oridonin is a covalent NLRP3 inhibitor with strong anti-inflammasome activity. Nat Commun. 2018;9.

162. Marchetti C, Swartzwelter B, Gamboni F, et al. OLT1177, a beta-sulfonyl nitrile compound, safe in humans, inhibits the NLRP3 inflammasome and reverses the metabolic cost of inflammation. Proc Natl Acad Sci U S A. 2018;115(7):E1530E1539.

163. Marchetti C, Swartzwelter B, Koenders MI, et al. NLRP3 inflammasome inhibitor OLT1177 suppresses joint inflammation in murine models of acute arthritis. Arthritis Res Ther. 2018;20(1):169.

164. Lamkanfi M, Mueller JL, Vitari AC, et al. Glyburide inhibits the Cryopyrin/Nalp3 inflammasome. J Cell Biol. 2009;187(1):61-70.

165. Cocco M, Pellegrini C, Martinez-Banaclocha H, et al. Development of an Acrylate Derivative Targeting the NLRP3 Inflammasome for the Treatment of Inflammatory Bowel Disease. J Med Chem. 2017;60(9):3656-3671.

166. Shim DW, Shin WY, Yu SH, et al. BOT-4-one attenuates NLRP3 inflammasome activation: NLRP3 alkylation leading to the regulation of its ATPase activity and ubiquitination. Sci Rep. 2017;7(1):15020.

167. He Y, Varadarajan S, Munoz-Planillo R, Burberry A, Nakamura Y, Nunez G. 3,4methylenedioxy-beta-nitrostyrene inhibits NLRP3 inflammasome activation by blocking assembly of the inflammasome. J Biol Chem. 2014;289(2):1142-1150.

168. Juliana C, Fernandes-Alnemri T, Wu J, et al. Anti-inflammatory compounds parthenolide and Bay 11-7082 are direct inhibitors of the inflammasome. J Biol Chem. 2010;285(13):9792-9802. 
NLRP3 inflammasome

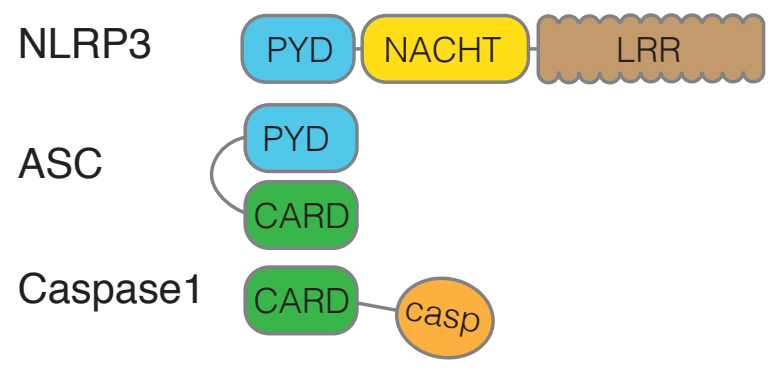

NLRP1 inflammasome

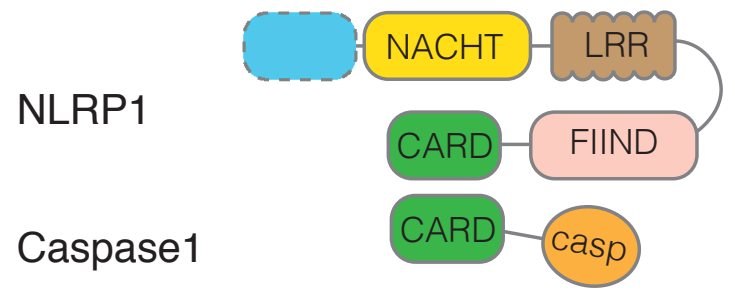

NLRC4 inflammasome

NLRC4

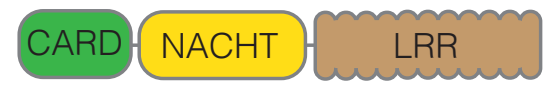

Caspase1

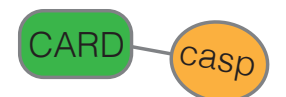

Pyrin inflammasome

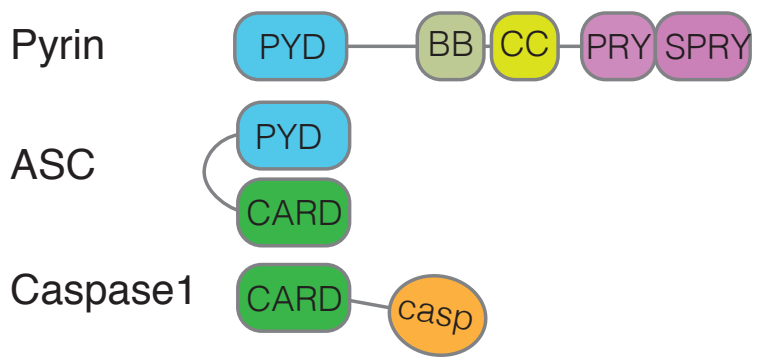

AIM2 inflammasome

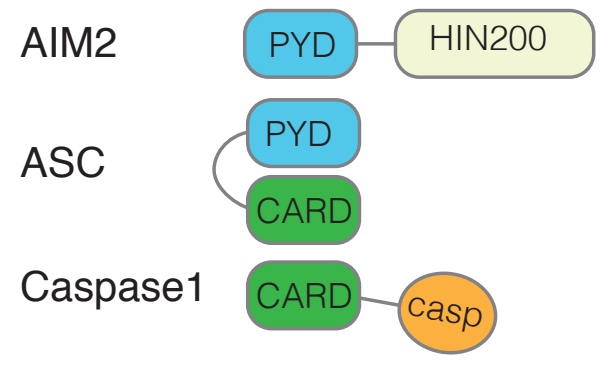




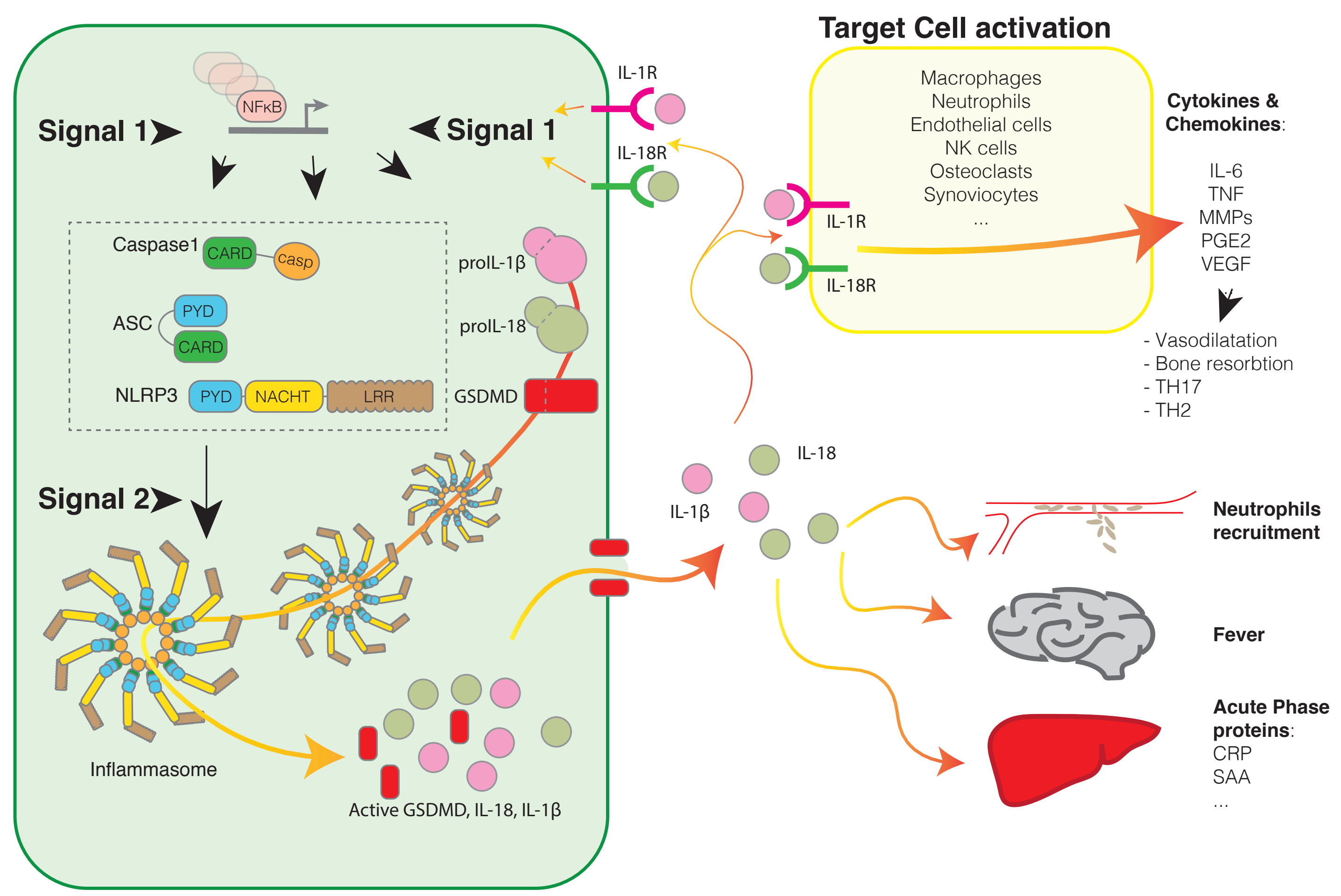




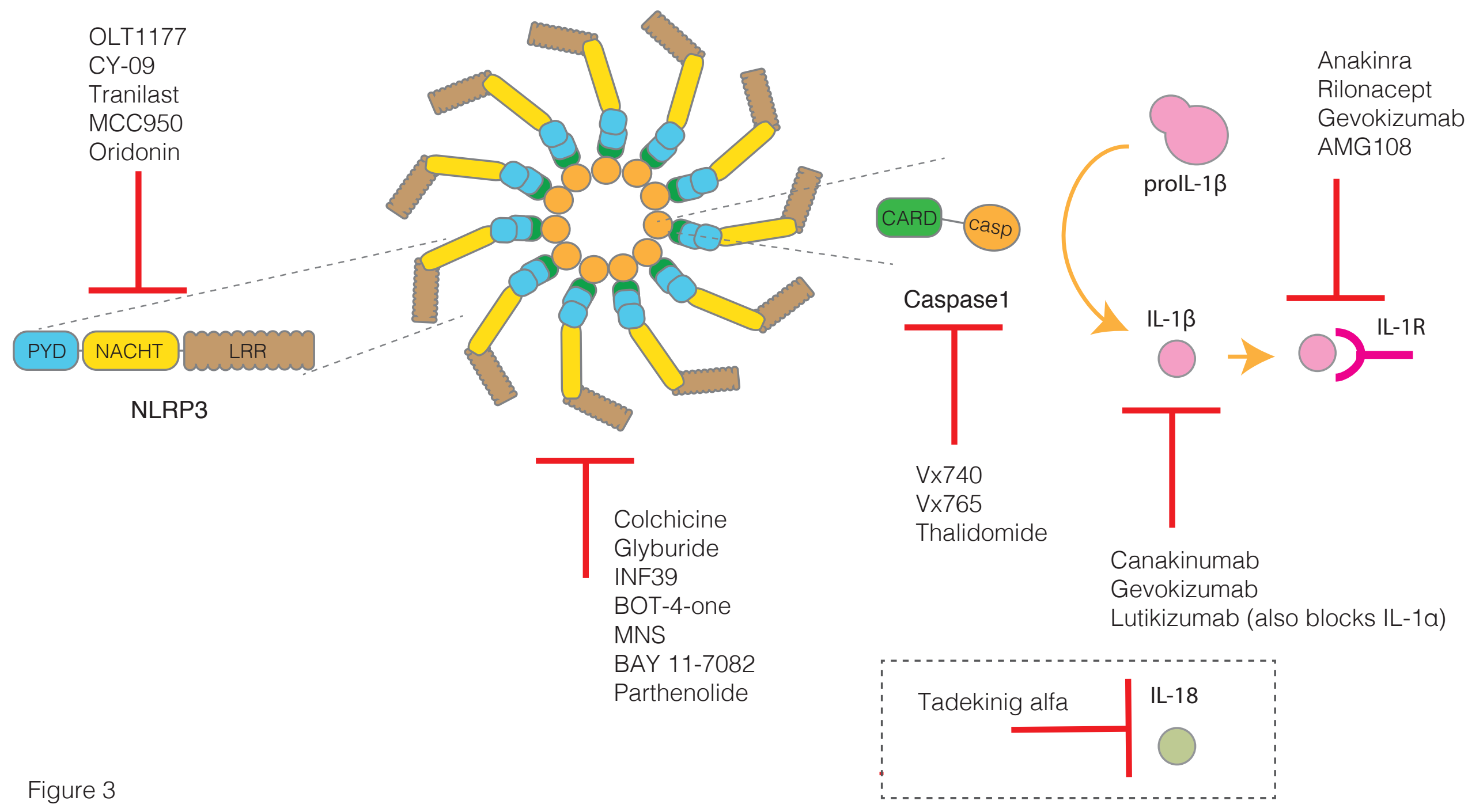

